\title{
Photon interference x-ray absorption fine structure
}

\author{
Y. Nishino, ${ }^{*}$ L. Tröger, P. Korecki, ${ }^{\dagger}$ and G. Materlik \\ Hamburger Synchrotronstrahlungslabor HASYLAB at Deutsches Elektronen-Synchrotron DESY, Notkestrasse 85, \\ D-22607 Hamburg, Germany
}

(Received 9 August 2001; published 18 October 2001)

\begin{abstract}
We report about a fine structure in $\mathrm{x}$-ray absorption spectra, named photon interference $\mathrm{x}$-ray absorption fine structure $(\pi \mathrm{XAFS})$. $\pi$ XAFS is due to the interference of x-rays inside the sample and extends across absorption edges. Using a platinum foil $\pi$ XAFS was measured in a high precision absorption experiment. Excellent agreement with a theoretical $\pi$ XAFS simulation is achieved in the energy range for above an absorption edge where photoelectron XAFS is negligible. $\pi$ XAFS provides a method for determination of atomic structure on short- and long-range order scale.
\end{abstract}

DOI: 10.1103/PhysRevB.64.201101

PACS number(s): 78.70.Dm, 07.85.-m, 61.10.Ht

For photon energies up to several ten keV, x-ray absorption is for heavier elements dominantly caused by the photoionization process. Absorption edges occur as sudden jumps in the spectra, whenever the incident x-ray energy exceeds the binding energy of an inner electron of the absorbing atom. Near and above such absorption edges, the absorption coefficient $\mu$ exhibits an x-ray absorption fine structure (XAFS) induced by the electronic and geometrical environment of the absorbing atom. Especially the oscillatory fine structure in the region up to around $2000 \mathrm{eV}$ above an absorption edge called EXAFS (extended XAFS) is widely used as a tool for structure determination. ${ }^{1,2}$ It results from the interference of the excited outgoing photoelectron wave with its backscattered waves from neighboring atoms. In conventional formalism, the initial-state photon is assumed to be a plane wave. EXAFS measures the radial distribution of neighboring atoms, coordination numbers, and averaged phonon properties on a short-range order scale.

In this paper, we discuss a fine structure in the x-ray absorption spectra which we call photon interference x-ray absorption fine structure ( $\pi$ XAFS). In $\pi$ XAFS, the initial-state photon is an interference field resulting from the incident photon wave and its coherently scattered waves from neighboring atoms (see Fig. 1 inset). $\pi$ XAFS extends in a wide photon energy range below and above absorption edges. It is shown that $\pi$ XAFS carries information about the short-range order structure of the sample comparable to EXAFS. Due to a long mean-free path of a photon, $\pi$ XAFS is also sensitive to longer-range order. $\pi$ XAFS and EXAFS are caused by modification of the absorption due to the multiatom effect. The importance of the multiatom effect in coherently excited resonant photoionization to yield near-neighbor information was also pointed out in Ref. 3 .

For a material with long-range order the photon interference effect is well known near a Bragg condition and results in a strong change in X-ray absorption. ${ }^{4}$ In this case the multiple scattered waves from many atoms interfere constructively and form a strong spatially modulated x-ray standing wave (XSW) field. By slightly changing the incident angle or energy, the XSW field moves in the sample, and accordingly the local $x$-ray intensity at the absorbing atom position changes. This effect has been intensively studied over narrow angle or energy ranges, and has been applied for structure determination. ${ }^{5} \mathrm{XSW}$ also explains structures in the absorption spectrum at Bragg conditions, which are known to complicate the XAFS analysis. ${ }^{6}$

Although never recognized so far in XAFS experiment and only mentioned theoretically recently, even far from Bragg conditions, the photon interference effect produces an observable oscillation in the extended absorption spectrum. ${ }^{7}$ The same photon interference effect is used in multipleenergy x-ray holography (MEXH) to obtain threedimensional atomic structure. ${ }^{8}$ Thus a careful measurement of the photon interference effect is needed even far from a Bragg condition, and as will be shown here opens the path to an experimental method.

If the sample does not have a preferred orientation of the neighbors, such as polycrystals, the photon interference effect is reduced by averaging over the neighbor orientations. Although the remaining oscillation amplitude is of the order of $10^{-4}$, it provides structural information as EXAFS does, and can be used as a structure determination method. State of the art XAFS beamline at synchrotron radiation source can be used to measure the effect even with required high preci-

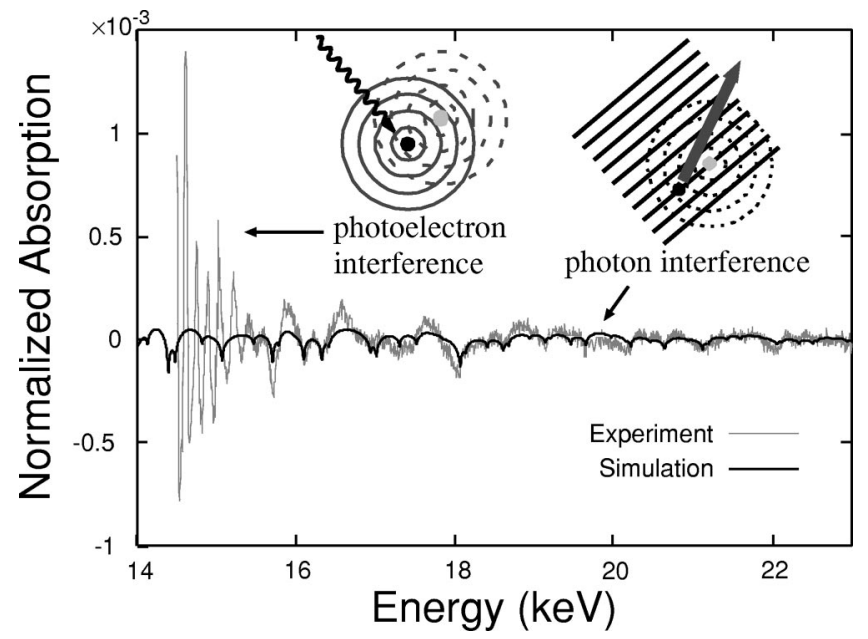

FIG. 1. Fine structure of the absorption coefficient of a Pt foil. The high-frequency oscillation dominating at energies lower than around $16 \mathrm{keV}$ is the Pt $L_{1}$ EXAFS contribution. Above $16 \mathrm{keV}$, the lower-frequency oscillation and the sharper structures are due to $\pi$ XAFS. 
sion in routine manner. We measured $\pi$ XAFS of a polycrystalline Pt foil using a high intensity, very stable synchrotron radiation beam. The experimental data of $\pi$ XAFS are in excellent agreement with the $\pi$ XAFS theory, and structural information is obtained with high precision.

In Ref. 7, the photoionization cross section for a sample with a preferred orientation is calculated in quantum electrodynamics (QED) including the initial-state photon interaction and the final-state photoelectron interaction with neighboring atoms. The photoionization total cross section $\sigma_{\mathrm{PI}}$, explaining MEXH as well as EXAFS, is given by

$$
\sigma_{\mathrm{PI}}=\sigma_{\mathrm{PI}, 0}\left[1+\chi^{\mathrm{x}}+\chi^{\mathrm{e}}\right],
$$

where $\sigma_{\mathrm{PI}, 0}$ represents the absorption of the isolated atom. $\chi^{\mathrm{X}}$ and $\chi^{\mathrm{e}}$ are oscillating functions ${ }^{9}$ in energy due to interference of x-rays and photoelectrons, respectively. The explicit expressions in the single-scattering plane wave approximation are given by

$$
\chi^{\mathrm{x}}=-2 r_{\mathrm{e}} \operatorname{Re} \sum_{l}^{\prime}\left[1-\left(\boldsymbol{\epsilon} \cdot \hat{\boldsymbol{r}}_{i}\right)^{2}\right] \frac{f_{i}^{\mathrm{x}}\left(\theta_{i}\right)}{r_{i}} e^{i\left(k_{\mathrm{x}} r_{i}+\boldsymbol{k}_{\mathrm{x}} \cdot \boldsymbol{r}_{i}\right)},
$$

and

$$
\chi^{\mathrm{e}}=-\frac{3}{k_{\mathrm{e}}} \sum_{l}{ }^{\prime} \frac{\left(\boldsymbol{\epsilon} \cdot \hat{\boldsymbol{r}}_{i}\right)^{2}}{r_{i}^{2}} \operatorname{Im}\left[f_{i}^{\mathrm{e}}(\pi) e^{2 i k_{\mathrm{e}} r_{i}}\right],
$$

where $\boldsymbol{k}_{\mathrm{x}}=k_{\mathrm{x}} \hat{\boldsymbol{k}}_{\mathrm{x}}$, and $\boldsymbol{\epsilon}$ are incident photon wave vector and polarization vector, respectively. $r_{\mathrm{e}}$ the classical electron radius. $k_{\mathrm{e}}$ denotes the size of photoelectron wave vector. The summations over $i$ are taken over neighboring atoms located at $\boldsymbol{r}_{i}=r_{i} \hat{\boldsymbol{r}}_{i}$ measured from the absorbing atom. $f_{i}^{\mathrm{x}}$ is the atomic form factor of the photon scattering, $f_{i}^{\mathrm{e}}$ is the scattering amplitude of the electron scattering from the $i$ th atom. $\pi$ in the argument of the electron-atom scattering amplitude $f_{i}^{\mathrm{e}}$ denotes the backscattering geometry. The photon interference contribution $\chi^{\mathrm{x}}$ of Eq. (2) is a known formula for MEXH. ${ }^{7,10}$ The photoelectron interference contribution $\chi^{\mathrm{e}}$ of Eq. (3) is the leading (single-scattering) contribution to EXAFS. ${ }^{2}$ Although the photon interference effect $\chi^{\mathrm{x}}$ has not been considered in present EXAFS theory, it gives a nonnegligible contribution. Similarly, the photoelectron interference contribution $\chi^{\mathrm{e}}$ should be taken into account in MEXH analysis. ${ }^{7}$ For a perfect crystal near a Bragg condition, the single scattering approximation in Eq. (2) is no longer appropriate because of strong multiple scattering. Dynamical theory of x-ray diffraction becomes necessary.

If a sample is polycrystalline, powder, amorphous, or nonsolid, the photoionization total cross section Eq. (1) needs to be averaged over the neighbor orientations $\hat{\boldsymbol{r}}_{i}$. The neighbor orientation random average $\bar{\chi}^{\mathrm{e}}$ of the photoelectron interference contribution $\chi^{\mathrm{e}}$ is simply given by Eq. (3) with replacing the factor $\left(\boldsymbol{\epsilon} \cdot \hat{\boldsymbol{r}}_{i}\right)^{2}$ by $1 / 3$. The $\hat{\boldsymbol{r}}_{i}$ random average of the photon interference contribution $\chi^{\mathrm{x}}$ is obtained by using a similar calculation procedure as the EXAFS calculations in Ref. 11, and one obtains

$$
\bar{\chi}^{\mathrm{x}}=-\frac{r_{\mathrm{e}}}{k_{\mathrm{x}}} \sum_{i \neq 0} \frac{1}{r_{i}^{2}} \operatorname{Im}\left[f_{i}^{\mathrm{x}}(\pi) e^{2 i k_{\mathrm{x}} r_{i}}\right] .
$$

After angular average, only backward scattering contributions remain. $\bar{\chi}^{\mathrm{x}}$ oscillates with the phase $2 k_{\mathrm{x}} r_{i}$. In Eq. (4), we have neglected higher-order terms in $\left(k_{\mathrm{x}} r_{i}\right)^{-1}$ by assuming that the incident $x$-ray wavelength is smaller than the inter-atomic distances. The x-ray polarization $\boldsymbol{\epsilon}$ dependent term in Eq. (2) results in higher-order terms in $\left(k_{\mathrm{x}} r_{i}\right)^{-1}$ after $\hat{\boldsymbol{r}}_{i}$ average.

$\pi$ XAFS $\bar{\chi}^{\mathrm{x}}$ of Eq. (4) and EXAFS $\bar{\chi}^{\mathrm{e}}$ are of equivalent form. It is important to note that the $\pi$ XAFS oscillation $\bar{\chi}^{\mathrm{x}}$ is periodic in photon wave number $k_{\mathrm{x}}$, whereas the EXAFS oscillation $\bar{\chi}^{\mathrm{e}}$ is periodic in photoelectron wave number $k_{\mathrm{e}}$. Relative amplitudes of both effects measured at identical $k$ values for the same material are $\bar{\chi}^{\mathrm{x}} / \bar{\chi}^{\mathrm{e}}=r_{\mathrm{e}} f^{\mathrm{x}} / f^{\mathrm{e}}$. For example, for Pt this ratio is $1.0 \times 10^{-3}$ at $k \approx 12 \AA^{-1} \cdot \bar{\chi}^{\mathrm{x}}$ is periodic also in the incident x-ray energy $\left(E_{x}=\hbar c k_{x}\right)$. The frequency of $\bar{\chi}^{\mathrm{x}}$ depends on the distance of an atom from the absorbing atom. The slowest oscillation is obtained for the nearest-neighbor distance (e.g., periodicity $2.4 \mathrm{keV}$ for a typical bond length of $2.6 \AA$ ).

Because $\chi^{\mathrm{x}}$ is the three-dimensional holographic signal of MEXH, $\bar{\chi}^{\mathrm{x}}$ is considered as a one-dimensional (angular averaged) $\mathrm{x}$-ray hologram. Similarly, EXAFS is regarded as an angular-integrated one-dimensional photoelectron hologram. ${ }^{7}$

For a realistic simulation of the angular averaged $\pi$ XAFS of Eq. (4), we additionally take into consideration the DebyeWaller factor $\exp \left(-2 \sigma^{2} k_{\mathrm{x}}^{2}\right)$ and the attenuation factor $\exp \left[-2 r_{i} / l_{\mathrm{x}}\left(E_{\mathrm{x}}\right)\right]$. Here, $\sigma^{2}$ is the mean-square relative displacement of atoms, and $l_{\mathrm{x}}\left(E_{\mathrm{x}}\right)$ is the photon mean free path. In EXAFS, the wave number of the photoelectron increases rapidly with the incident $\mathrm{x}$-ray energy as $k_{e}$ $=\sqrt{2 m_{\mathrm{e}}\left(E_{\mathrm{x}}-E_{\mathrm{b}}\right)} / \hbar$, where $E_{\mathrm{b}}$ is the binding energy of the core electron and $m_{\mathrm{e}}$ is the electron mass. Therefore, the EXAFS Debye-Waller factor decreases rapidly with incident energy. Consequently, the EXAFS signal can be observed relatively close to absorption edges. For $\pi \mathrm{XAFS}$, the wave number of $x$-rays increases slowly with the incident energy as $k_{\mathrm{x}}=E_{\mathrm{x}} /(\hbar c)$, thus $\pi \mathrm{XAFS}$ extends over a wide range of energy.

The $\pi$ XAFS signal was measured at the bending magnet beamline X1 at the Hamburger Synchrotronstrahlungslabor HASYLAB at Deutsches Elektronen-Synchrotron DESY. This beamline is optimized for high-precision x-ray absorption experiments. The $\mathrm{x}$-rays were monochromatized by a $\mathrm{Si}(311)$ double-crystal monochromator which was stabilized using a remotely controlled, digital monochromator stabilization. An optical encoder system precisely measures the monochromator crystal angle determining the incident $\mathrm{x}$-ray energy. A high purity (>99.99\%) $25 \mu \mathrm{m}$ thick Pt foil was used which ensures that the signal of the order of $10^{-4}$ is not influenced by impurities. The incident energy was varied from $14.5 \mathrm{keV}$ to $22.0 \mathrm{keV}$ in $3 \mathrm{eV}$ steps over a wide region above the Pt $L_{1}$ absorption edge. A wide energy range is 


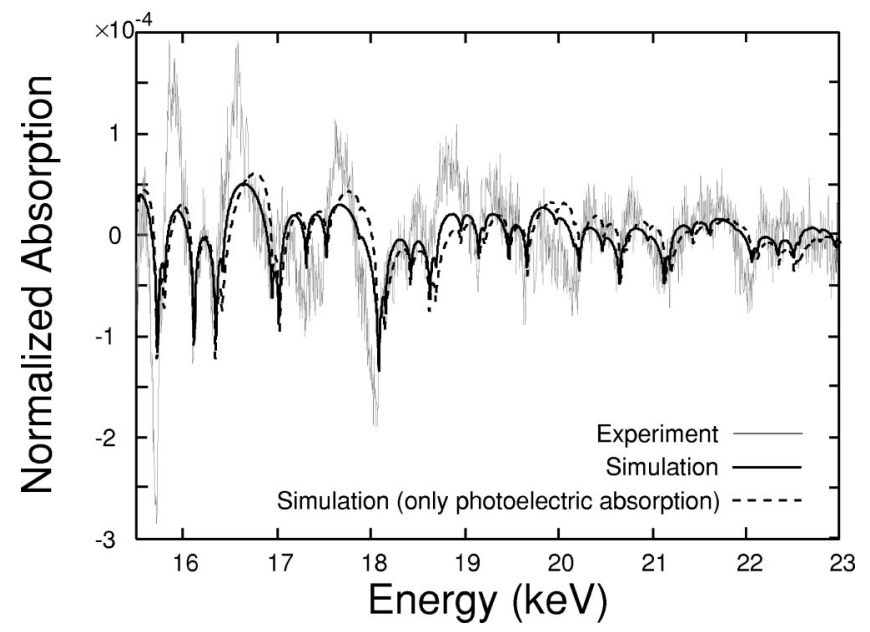

FIG. 2. $\pi$ XAFS of a Pt foil. Enlargement of Fig. 1.

necessary to measure slow oscillatory contributions of $\pi$ XAFS. The beam size at the sample position was $10 \mathrm{~mm}$ in horizontal and $1.5 \mathrm{~mm}$ in vertical direction. The photon flux on the sample was around $10^{10} \mathrm{~s}^{-1}$. Measurements were performed in transmission mode at room temperature. The intensities of incident and transmitted x-rays were measured by flow ionization chambers using $\mathrm{Ar}$ and $\mathrm{Kr}$ at atmospheric pressure. The absorption coefficient $\mu$ is determined from the incident $I_{0}$ and transmitted $I_{\mathrm{t}}$ intensities using the relation: $\mu d=-\ln \left[\left(I_{\mathrm{t}} / I_{0}\right) /\left(I_{\mathrm{t}, \mathrm{e}} / I_{0, \mathrm{e}}\right)\right]$, where $d$ is the sample thickness and the subscripts e denote measurements without the Pt sample to subtract all contributions other than from the sample.

$\mu d$ was fitted by a Victoreen function in order to subtract the isolated-atom contribution $\mu_{0} d$. Fitting was performed in $2 \mathrm{keV}$ or wider energy ranges. The normalized signal $(\mu$ $\left.-\mu_{0}\right) / \mu_{0}$ connects smoothly between subsequent energy ranges of the fitting procedure. Using a test signal, this fitting procedure was shown to be appropriate although the lowest frequency mode of the test signal is partly lost.

Figure 1 shows the normalized absorption coefficient. The high-frequency Pt $L_{1}$ EXAFS signal fades out as the incident energy increases, and the fine structure of $\pi$ XAFS becomes dominant in the energy region of around $16 \mathrm{keV}$ and above. Figure 2 shows the detailed structure of $\pi$ XAFS in the energy region from 15.5 to $23 \mathrm{keV}$. Together with the experimental signal, simulations using Eq. (3) with and without the additional contribution of the elastic scattering (photons scattered off the sample) are shown. Inclusion of the elastic scattering only slightly changes the theoretical estimation. The measured signal shows an excellent agreement with the simulation. Compared to EXAFS, $\pi$ XAFS has less contributions from phase shift and multiple scattering which makes its simulation much simpler. The cluster for the simulation had a radius of $600 \AA$. For simplicity, correlated atomic motion was neglected, and for the mean-square relative displacements $\sigma^{2}$ twice the value in X-ray diffraction ${ }^{12}$ was assumed.

Although the nearest-neighbor contribution to $\pi$ XAFS is of low frequency, the $\pi$ XAFS signal in Fig. 2 contains sharp negative peaks because of large contributions from far coor-

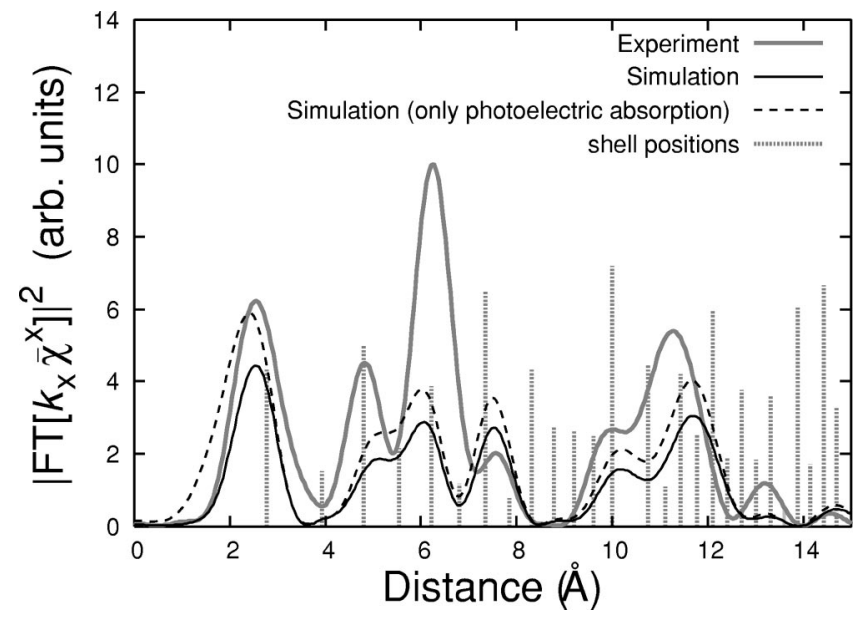

FIG. 3. Fourier transform of $\pi$ XAFS of a Pt foil. The Fourier transformation was performed for the signal $k_{\mathrm{x}} \bar{\chi}^{\mathrm{x}}$ with respect to $2 k_{\mathrm{x}}$ in the $k_{\mathrm{x}}$ range of 8.1 to $11.15 \AA^{-1}$ (energy range 16 to 22 $\mathrm{keV}$ ). The modulo square values of the Fourier transform are shown. Vertical lines indicate the positions of Pt coordination shells. Their height corresponds to their (weighted) coordination numbers. The peak width is determined by the $k_{\mathrm{x}}$ range.

dination shells. It is important to note that the positions of the characteristic sharp structure in $\pi$ XAFS coincide with the fulfillment of $\pi / 2$ Bragg condition, i.e., backscattering of the incident $\mathrm{X}$-ray beam. The sharp negative peaks in $\pi$ XAFS are understood in the following way. Due to XSW formation, a lattice plane at an incident angle slightly larger than the Bragg angle $\theta_{B}$ absorbs more x rays than one at an incident angle slightly less than $\theta_{B}$. In a polycrystalline sample for $\theta_{B} \neq \pi / 2$, contributions of lattice planes with different orientations average this difference. Compared to this averaged absorption for $\theta_{B} \neq \pi / 2$, less $\mathrm{x}$ rays are absorbed at $\theta_{B}=\pi / 2$, because incident angles larger than $\pi / 2$ and, consequently, lattice planes with higher absorption than the average do not exist. In the conventional XSW measurement, a crystalline sample with mosaic spread less than the width of the Bragg reflection is necessary. In Refs. 6 and 13 it was shown that an energy scanned XSW measurement at $\theta_{B}$ $=\pi / 2$ is much less sensitive to imperfections of the crystal because of a wide (of order $1^{\circ}$ ) Bragg reflection width. ${ }^{14}$ Here it is shown that the energy dependence of the x-ray absorption at $\theta_{B}=\pi / 2$ is also measured in polycrystalline samples. The elastic scattering is expected to produce a step structure at the same energy position because of appearance of a new Debye-Scherrer cone. It is especially important below the absorption edge, and peaks with asymmetric shape are observed in an additional absorption measurement below the $L$ edge for $\mathrm{Pt}$ and $\mathrm{W}$ powders.

Figure 3 shows the Fourier transform of $k_{\mathrm{x}} \bar{\chi}^{\mathrm{x}}$ with respect to $2 k_{\mathrm{x}}$. According to Eq. (4), peaks corresponding to radial coordinates of neighbor atoms are expected. This is analogous to the case of EXAFS where a Fourier transform of $\bar{\chi}^{\mathrm{e}}$ (or $\chi^{\mathrm{e}}$ ) with respect to $2 k_{\mathrm{e}}$ shows atomic peaks. The peak positions of the experimental data in Fig. 3 agree well with those from the simulation up to radial distance of $15 \AA$. Figure 3 demonstrates that $\pi$ XAFS provides direct short- 
range order structural information. Already in this first experiment the agreement of inter-atomic distances in experiment and simulation with accuracy better than $0.1 \AA$ is achieved. Accuracy will be improved by increasing the data energy range. The accuracy of $\pi$ XAFS with respect to the determination of atomic short-range order is comparable to an EXAFS measurement of corresponding $k$ range. Even with the elastic scatting contribution the Fourier analysis is fully valid, because it turned out that the Fourier transform of the structure in the elastic scattering contribution also provides peaks corresponding to the atomic coordination shells. The detailed connection to the elastic scattering will be described in a separate paper.

$\pi \mathrm{XAFS}$ bears importance for the XAFS analysis, because $\pi$ XAFS exists also in the XAFS energy region. Reliable background subtraction is especially important in the EXAFS analysis. Slow oscillations in EXAFS data lead to a peak in the Fourier transform well below the nearestneighbor distance. Scattering on the atomic potential of the absorbing atom or interstitial charge density between atoms was discussed to induce related variations in the absorption coefficient (atomic XAFS).$^{15}$ Multielectron photoexcitations as well occur in the absorption spectrum showing sharp edges or broader structures. ${ }^{16} \pi$ XAFS exists in the same energy range, therefore it will be important to disentangle all effects carefully.

Since the $\pi$ XAFS oscillations are a function of photon wave vector $k_{\mathrm{x}}$ (or incident energy $E_{\mathrm{x}}$ ), at high energies such as used here a small change in atomic structure results in strong variations. A change in the lattice constant $a$ by, e.g., $\Delta a=1.0 \times 10^{-3} \AA$ induces a shift of a peak position by $\Delta E_{\mathrm{x}}=(\Delta a / a) E_{\mathrm{x}} \approx 5.0 \mathrm{eV}$ at $E_{\mathrm{x}} \approx 20 \mathrm{keV}$ for $\mathrm{Pt}(a$ $=3.92 \AA$ ). With respect to long-range order structure determination, a $\pi$ XAFS accuracy is expected comparable to diffraction techniques.

In summary, we have shown the existence of a fine structure in the $\mathrm{x}$-ray absorption spectrum called $\pi$ XAFS which is due to photon interference effects. The first analysis of $\pi$ XAFS was performed for a Pt foil. The $\pi$ XAFS experiment agrees excellently with a simple theoretical simulation. Experimentally it was shown that an x-ray absorption spectrum far above the absorption edge is useful to study shortand long-range order complementary to EXAFS and diffraction techniques. Although the modulation is small in samples without preferred orientation, modern synchrotron radiation beamlines can be used routinely to measure the effect. By using other detection techniques like the one of characteristic $\mathrm{x}$-ray fluorescence, $\pi$ XAFS experiments can be performed in an element sensitive manner. ${ }^{17}$
*Present address: SPring-8/Harima Institute, RIKEN, Mikazukicho, Sayo-gun, Hyogo 679-5148, Japan.

${ }^{\dagger}$ Present address: Institute of Physics, Jagiellonian University Reymonta 4, 30-059 Krakow, Poland.

${ }^{1}$ de L.R. Kronig, Z. Phys. 70, 317 (1931).

${ }^{2} X$-Ray Absorption: Principles, Applications, Techniques of EX$A F S, S E X A F S$ and XANES, edited by R. Prins and D.C. Koningsberger (Wiley, New York, 1988), and references therein.

${ }^{3}$ A. Kay et al., Science 281, 679 (1998).

${ }^{4}$ B.W. Batterman and H. Cole, Rev. Mod. Phys. 36, 681 (1964).

${ }^{5}$ M.J. Bedzyk and G. Materlik, Phys. Rev. B 32, 6456 (1985); J. Zegenhagen, Surf. Sci. 18, 199 (1993), and references therein.

${ }^{6}$ T. Ohta et al., Jpn. J. Appl. Phys., Part 2 24, L475 (1985).

${ }^{7}$ Y. Nishino and G. Materlik, Phys. Rev. B 60, 15074 (1999); 61, 14 845(E) (2000).

${ }^{8}$ T. Gog et al., Phys. Rev. Lett. 76, 3132 (1996).

${ }^{9}$ Note that $\bar{\chi}^{\mathrm{x}}$ and $\bar{\chi}^{\mathrm{e}}$ in Ref. 7 are respectively equivalent with $-\chi^{\mathrm{x}}$ and $-\chi^{\mathrm{e}}$ in this work.

${ }^{10}$ B. Adams et al., Phys. Rev. B 57, 7526 (1998).
${ }^{11}$ P.A. Lee, Phys. Rev. B 13, 5261 (1976).

${ }^{12}$ International Tables for X-ray Crystallography, edited by C.H. Macgillavry and G.D. Rieck (Kluwer Academic, Dordrecht, 1985), Vol III.

${ }^{13}$ T. Ohta et al., Nucl. Instrum. Methods Phys. Res. A 246, 760 (1986); Phys. Rev. Lett. 58, 1460 (1987).

${ }^{14}$ K. Kohra and T. Matsushita, Z. Naturforsch. Teil A 27, 484 (1972); W. Graeff and G. Materlik, Nucl. Instrum. Methods Phys. Res. 195, 97 (1982); A. Caticha and S. Caticha-Ellis, Phys. Rev. B 25, 971 (1982).

${ }^{15}$ B.W. Holland et al., J. Phys. C 11, 633 (1978); J.J. Rehr et al., Phys. Rev. B 49, 12347 (1994).

${ }^{16}$ H.W. Schnopper, Phys. Rev. 131, 2558 (1963); E.A. Stern, Phys. Rev. Lett. 49, 1353 (1982); A. Filipponi, E. Bernieri, and S. Mobilio, Phys. Rev. B 38, 3298 (1988); A. Kodre et al., Phys. Rev. A 45, 4682 (1992).

${ }^{17}$ After submission of this paper our attention was drawn to footnote 32 in G.A. Miller and L.B. Sorensen, Phys. Rev. B 56, 2399 (1997) by its authors. 